\title{
Selective destruction of viruses with ultrashort pulsed lasers
}

Kong-Thon Tsen, Shaw-Wei Tsen, T. C. Wu, Karen Kibler, and Bert Jacobs

A new method for killing viral particles may have important medical applications like disinfecting blood components and treating diseases.

A variety of techniques can kill unwanted viral particles. While UV disinfection ${ }^{1,2}$ is effective, for example, UV lamps target both nuclei acids and proteins, so they damage not only the viral particles, but also mammalian cells. UV irradiation also raises concerns of mutation and has shadowing effects, or decreases in penetration length caused by the viral nucleic acids and protein shells. Similarly, microwave absorption is not effective because most of the energy is transferred to water and not the viral particle. Recently, a photochemical technique ${ }^{3}$ was developed to disinfect blood supplies. However, potential risks and side effects have hindered its application. A new method that circumvents these problems is needed.

We have developed a photonic approach for selectively inactivating viruses. Our method uses a near-IR ultrashort pulsed (USP) subpicosecond fiber laser system instead of UV lamps. It targets only the weak links on the protein shells of viral particles. By tuning to the appropriate laser power density, we demonstrate that it is feasible to damage the protein shells, leading to their inactivation without harming mammalian cells. Specifically, we show that this method can selectively inactivate viral particles ranging from non-pathogenic viruses such as M13 bacteriophage and tobacco mosaic virus (TMV) to pathogenic viruses like human papillomavirus (HPV) and human immunodeficiency virus (HIV). At the same time, it leaves sensitive materials like human Jurkat T cells, human red blood cells, and mouse dendritic cells unharmed.

Any mechanical object has normal modes in which it oscillates around its equilibrium geometry. By choosing the pulse duration of the visible/near-IR laser to be shorter than or near to the normal mode's oscillation period, the laser pulse in a singlebeam excitation configuration has significant spectral content at the resonant frequency. This can bring a macro-particle such as a virus into oscillation through impulsive stimulated Raman
(A)

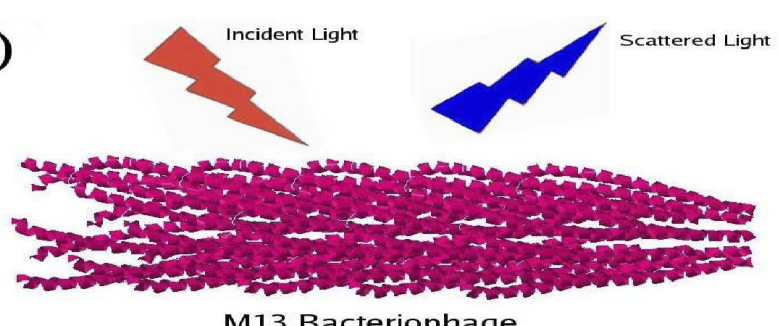

(B)

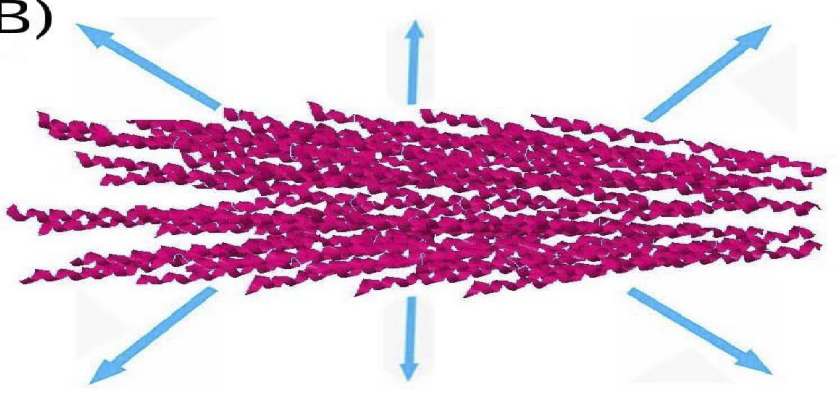

(C)

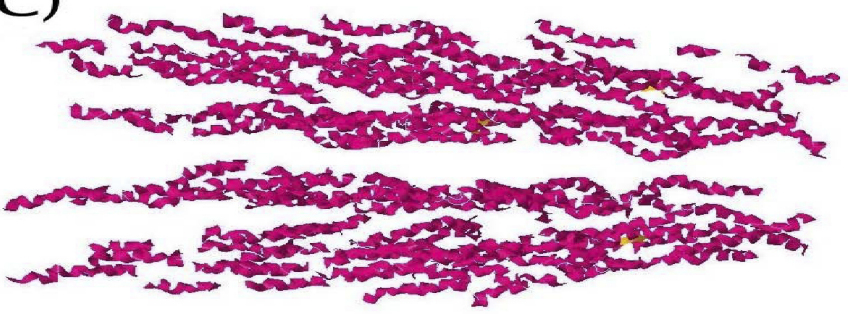

Figure 1. Demonstration of how a M13 bacteriophage is inactivated by a USP laser. (A) The laser interacts with the viral particle. (B) The laser excites the virus' protein shell by ISRS. (C) When the excitation's amplitude is large enough, the protein shell breaks apart, inactivating the viral particle.

scattering (ISRS). ${ }^{4-7}$ Figure 1 shows how the macro-particle, M13 bacteriophage, is inactivated through ISRS by a USP laser. An ultrashort pulse excites the vibrational modes on the protective shell to such high energy states that it breaks off the weak links 
Table 1. Threshold laser power density for inactivation of various viruses and mammalian cells.

\begin{tabular}{|l|l|l|l|l|l|l|l|}
\hline & M13 & TMV & HPV & HIV & $\begin{array}{l}\text { Human } \\
\text { Red } \\
\text { Blood } \\
\text { Cell }\end{array}$ & $\begin{array}{l}\text { Human } \\
\text { Jurkat } \\
\text { T cell }\end{array}$ & $\begin{array}{l}\text { Mouse } \\
\text { Dendritic } \\
\text { Cell }\end{array}$ \\
\cline { 2 - 7 } & 0.06 & 0.85 & 1.0 & 1.1 & 15 & 22 & 12 \\
\hline $\begin{array}{l}\text { Threshold } \\
\text { power } \\
\text { density for } \\
\text { inactivation } \\
\left(\mathrm{GW} / \mathrm{cm}^{2}\right)\end{array}$ & 0.0 & & & & & \\
\hline
\end{tabular}

on the shell, damaging the shell and leading to inactivation of the M13 bacteriophage.

Table 1 summarizes the threshold laser power density for inactivation of M13 bacteriophage, TMV, HPV, HIV, human red blood cells, human Jurkat cells, and mouse dendritic cells with a USP fiber laser operating at $\lambda=776 \mathrm{~nm}$. Many viruses can be inactivated with a laser power density of about $1 \mathrm{GW} / \mathrm{cm}^{2}$. On the other hand, much higher laser power intensities of $\geq$ $10 \mathrm{GW} / \mathrm{cm}^{2}$ are necessary to inactivate mammalian cells. These results indicate that there is a window in laser intensity bounded approximately by $1 \mathrm{GW} / \mathrm{cm}^{2}$ and $10 \mathrm{GW} / \mathrm{cm}^{2}$ that allows us to inactivate unwanted microorganisms such as viruses while leaving useful materials such as mammalian cells unharmed. It is therefore plausible that the USP laser, if appropriately manipulated, can be used to selectively kill blood-borne pathogens with minimal damage to sensitive materials. It is this selectivity that distinguishes our approach.

One likely reason for the selective killing of microorganisms demonstrated in Table 1 is the difference in their structure. We know the cell's lipid membrane structure is significantly different from that of the viral particle's capsid. Another likely reason is the size differential. Viral particles are typically much smaller than mammalian cells. For example, HIV is an enveloped virus with a capsid and is about $0.1 \mu \mathrm{m}$ in diameter, whereas the shape of a human red blood cell is like a doughnut, about $10 \mu \mathrm{m}$ in diameter and $2 \mu \mathrm{m}$ thick. The mouse dendritic cell is about $10 \mu \mathrm{m}$ in diameter. Since the viruses and cells are embedded in water, the water molecules play a crucial role in damping the vibrations excited by the laser. The relatively large size of either the human red blood cell or the mouse dendritic cell compared with that of the viral particle means there are more water molecules surrounding the red blood cells and dendritic cells than HIV. In other words, the damping associated with the coherent/incoherent excitation the laser creates is less for HIV than for red blood cells or dendritic cells. As a result, the vibration amplitude created by a given laser power density can be much higher for HIV than for red blood cells or mouse dendritic cells. Testing for these possible scenarios is currently underway.

The USP laser technology targets the global mechanical properties of the viral protein shell, making it relatively insensitive to local genetic mutation in target viruses. As a result, the approach can inactivate both the wild and mutated strains of viruses. This intriguing property is particularly important in the treatment of diseases involving rapidly mutating viral species like HIV. Our USP laser approach could be used for disinfecting viral pathogens in blood products and for the treatment of blood-borne viral diseases in the clinic.

Kong-Thon Tsen would like to thank Raydiance Inc. for the loan of a USP fiber laser.

\section{Author Information}

\section{Kong-Thon Tsen}

Department of Physics

Arizona State University (ASU)

Tempe, AZ

Kong-Thon Tsen's research focuses on the interaction of light in solid-state and biological systems, in particular on the use of ultrafast laser sources to elucidate novel electron transport phenomena, dynamic properties of lattice vibrations in nanostructure semiconductors, and microscopic mechanisms in biological 
systems such as viruses, bacteria, and cells. He has more than 170 refereed publications and has edited four books about ultrafast phenomena.

\section{Shaw-Wei Tsen}

Washington University School of Medicine

St. Louis, MO

\section{T. C. Wu}

Pathology Laboratory

Johns Hopkins Medical Institutions

Baltimore, MD

\section{Karen Kibler and Bert Jacobs}

Biodesign Institute

ASU

Tempe, AZ
References

1. K. Rosenheck and P. Doty, The far ultraviolet absorption spectra of polypeptide and protein solutions and their dependence on conformation, Proc. Natl. Acad. Sci. 47 (11), pp. 1775-1785, 1961.

2. J. C. Sutherland and K. P. Griffin, Absorption spectrum of DNA for wavelengths greater than 300nm, Radiation Res. 86, p. 3990410, 1981.

3. B. J. Bryant and H. G. Klein, Pathogen Inactivation: The Definitive Safeguard for the Blood Supply, Arch. Pathol. Lab. Med. 131, pp. 719-733, 2007.

4. Y.-X. Yan, E. B. Gamble Jr., and A. Keith Nelson, Impulsive stimulated scattering: general importance in femtosecond laser pulse interactions with matter, and spectroscopic applications, J. Chem. Phys. 83, pp. 5391-5399, 1985.

5. K. A. Nelson, R. J. D. Miller, D. R. Lutz, and M. D. Fayer, Optical generation of tunable ultrasonic waves, J. Appl. Phys. 53, pp. 1144-1149, 1982.

6. K. T. Tsen, S-W. D. Tsen, C-L. Chang, C-F. Hung, T. C. Wu, and J. G. Kiang, Inactivation of viruses by laser-driven coherent excitations via impulsive stimulated Raman scattering process, J. Biomed. Opt. 12 (064030), pp. 1-6, 2007.

7. K. T. Tsen, S-W. D. Tsen, O. F. Sankey, and J. G. Kiang, Selective inactivation of microorganisms with near-infrared femtosecond laser pulses, J. Phys. Cond. Matter 19, pp. 472201-1-472201-7, 2007. 